\title{
Exclusion in a priority queue
}

\author{
${ }^{1}$ Jan de Gier and ${ }^{2}$ Caley Finn \\ Department of Mathematics and Statistics, The University of Melbourne, 3010 VIC, \\ Australia \\ E-mail: ${ }^{1}$ jdgier@unimelb.edu.au, ${ }^{2}$ c.finn3@pgrad.unimelb.edu.au
}

\begin{abstract}
We introduce the prioritising exclusion process, a stochastic scheduling mechanism for a priority queueing system in which high priority customers gain advantage by overtaking low priority customers. The model is analogous to a totally asymmetric exclusion process with a dynamically varying lattice length. We calculate exact local density profiles for an unbounded queue by deriving domain wall dynamics from the microscopic transition rules. The structure of the unbounded queue carries over to bounded queues where, although no longer exact, we find the domain wall theory is in very good agreement with simulation results. Within this approximation we calculate average waiting times for queueing customers.
\end{abstract}




\section{Introduction}

In this work we introduce the prioritising exclusion process (PEP): a stochastic scheduling mechanism for a priority queue, where high priority customers overtake low priority customers in order to receive service sooner. The queue of customers is represented by a one dimensional lattice, which grows and shrinks as customers arrive and are served. Lattice sites are either empty or occupied by a single particle, representing low and high priority customers respectively; particles hop forwards stochastically into empty sites, corresponding to a high priority customer overtaking the low priority customer immediately ahead of them, but the exclusion rule prevents particles hopping into or over occupied sites. The PEP is closely related to a priority queuing model first introduced by Kleinrock [1], and the subject of more recent work [2]. Priority queueing systems are relevant in healthcare applications, both as a way to efficiently manage hospital queues with patients of differing urgency [2], and to describe actual practise in emergency rooms [3].

The hopping and exclusion in the PEP is analogous to that of the totally asymmetric simple exclusion process (TASEP) [4,5, which is one of the most thoroughly studied and central models of non-equilibrium statistical mechanics [6]. The TASEP is a microscopic model of a driven system [10], and has been the focus of much mathematical interest due to the fact that it is integrable. Many tools have been applied to or developed for the TASEP. Its exact stationary distribution is known 11, 12, and can be written in matrix product form [9, 13]. Its dynamic properties are studied by means of the Bethe ansatz [8,14, 15] and for the infinite lattice powerful techniques from random matrix theory are available [16. Domain wall theory [17] provides a phenomenological explanation of the stationary behaviour of the TASEP. Domain wall theory is amenable to generalisation to more complicated models that may not be integrable, and we will describe it in more detail later.

Recently, several generalisations of the TASEP have been proposed, which allow the lattice length to vary dynamically as is the case in the PEP. Such models are of theoretical interest in statistical physics as they are grand-canonical analogues of the TASEP. The review 18 highlights many biological applications, such as modelling filament growth 1921 and length regulation 22, 23]. There have been applications to queueing theory as well. The exclusive queueing process (EQP) 24,25] uses an exclusion process to model the motion of customers waiting in a queue. In the EQP, customers (all of a single priority class) are represented by particles, with empty lattice sites for the space between them. The hopping of particles represents customers shuffling forwards as space becomes available. Beyond applications, these models are also of interest because of the rich phase structure the varying lattice length introduces 26 29].

As is typical of a queueing system, the PEP has a phase transition from a phase with finite expected queue length, to one with an unbounded queue length increasing with time. This transition occurs when the rate of arrival of customers exceeds the service rate. In the latter case, we treat the lattice as infinite in length in order to study 
the late time limit. The PEP has a natural domain wall structure, and the domain wall dynamics can be derived directly from the microscopic transition rules, similarly to [30]. In the unbounded phase, in the infinite lattice limit, the solution of the domain wall equations gives exact local density profiles. The domain wall solution reveals a second phase transition where the 'jam' of high priority customers waiting at the service end becomes infinite.

When the expected lattice length remains finite (the bounded phase), the domain wall theory leads to approximate solutions only, but the structure from the unbounded phase carries over. We see a remnant of the jamming transition from the unbounded phase as a crossover where the jam of high priority customers at the service end delocalises, and the expected jam length becomes comparable to the queue length. Then by defining 'aggregated correlation functions', we find that the form of the unbounded solution can be applied in the bounded phase as an alternative to mean field theory, giving a very accurate calculation of customer waiting times.

\subsection{The model}

In the lattice bulk, the PEP behaves as a TASEP: sites are either occupied by a single particle or empty, and particles hop forwards into empty sites with rate $p$. At the boundaries the PEP differs from the TASEP. The PEP lattice can be extended on the left by the addition of a filled or empty lattice site, with rates $\lambda_{1}$ and $\lambda_{2}$ respectively. At the other boundary, the rightmost site is removed with rate $\mu$, irrespective of its occupation. These rules, summarised in Figure 1, allow both the lattice length and particle number to vary.

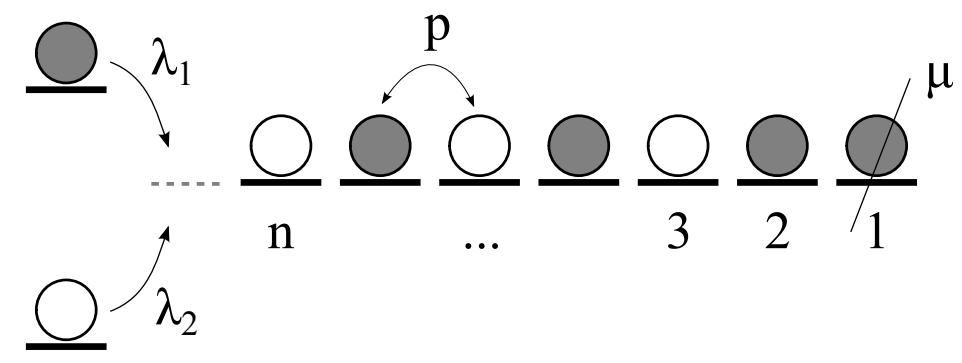

Figure 1. PEP transition rates, filled circles are occupied sites.

We specify a PEP configuration by binary variables $\tau_{i}$ with $\tau_{i}=1$ for a filled site and $\tau_{i}=0$ for an empty site. Usually we will number sites from right to left and write a length $n$ configuration as

$$
\boldsymbol{\tau}=\tau_{n} \tau_{n-1} \ldots \tau_{1}
$$

\subsection{A queueing system}

The PEP can be interpreted as a priority queueing system with two classes of customers. The lattice, itself, is the queue of customers, with filled sites representing high priority 
customers (class 1) and empty sites representing low priority customers (class 2). The rates $\lambda_{1}$ and $\lambda_{2}$ are the arrival rates of high and low priority customers respectively, and the rate $\mu$, is the rate at which customers are served and leave the queue.

In this interpretation, a particle hopping forward one site corresponds to a high priority customer stepping ahead of the low priority customer immediately in front of them. The stochastic overtaking is the scheduling mechanism in this priority queue, giving high priority customers preferential treatment over low priority. The larger the overtake rate $p$, the greater the advantage.

The PEP is modelled on a well studied priority queueing system introduced by Kleinrock [1, 31] and now known as the accumulating priority queue (APQ) [2]. In the $\mathrm{APQ}$, customers have a priority value which accumulates linearly with time. Class 1 customers accumulate priority faster than class 2, thus overtaking them in the service queue. The key difference between the APQ and the PEP is that, for a given sequence of arrivals, overtaking in the APQ is deterministic, but in the PEP the overtakes occur stochastically.

The PEP is also related to a simpler queueing system, the $M / M / 1$ queue (see, for example, [32]). The total arrival rate of customers to the PEP is $\lambda=\lambda_{1}+\lambda_{2}$, and the service rate is $\mu$. Both these rates are independent of the internal arrangement of the queue, and the prioritising parameter $p$. So, if we are interested only in the total length of the queue, we can treat the system as a $M / M / 1$ queue with arrival rate $\lambda$ and service rate $\mu$. The state of a $M / M / 1$ queue is characterised simply by the length, $n$, with probability distribution $P_{n}$ obeying the master equation

$$
\begin{aligned}
& \frac{\mathrm{d} P_{0}}{\mathrm{~d} t}=\mu P_{1}-\lambda P_{0} \\
& \frac{\mathrm{d} P_{n}}{\mathrm{~d} t}=\lambda P_{n-1}+\mu P_{n+1}-(\mu+\lambda) P_{n}, \quad n>0 .
\end{aligned}
$$

The stationary length distribution of the $M / M / 1$ queue (and hence for the PEP) is the solution of $\mathrm{d} P_{n} / \mathrm{d} t=0$, which is

$$
P_{n}=\left(1-\frac{\lambda}{\mu}\right)\left(\frac{\lambda}{\mu}\right)^{n},
$$

when $\lambda<\mu$, i.e. when the total arrival rate is less than the service rate. In this case the system is described as stable, because the queue length does not grow without bound. The expected queue length is finite, given by

$$
\langle n\rangle=\frac{\lambda}{\mu-\lambda} .
$$

We will call this the bounded phase of the PEP.

When $\lambda>\mu$, the system is unstable and the expected queue length grows as

$$
\langle n\rangle \sim(\lambda-\mu) t
$$

In the late time limit, we can treat the queue as infinite in length. We call this the unbounded phase of the PEP. 
At the special value $p=0$, the PEP really does reduce to a $M / M / 1$ queue. Customers arriving at rate $\lambda$ are high priority with probability $\lambda_{1} / \lambda$ or low priority with probability $\lambda_{2} / \lambda$. But as there is no overtaking, there is no reordering of customers, and no advantage in being a class 1 customer. The probability of high or low at any site is the same as at arrival, i.e. $\lambda_{1} / \lambda$ or $\lambda_{2} / \lambda$, respectively. In the bounded phase, the probability of the length $n$ configuration $\tau_{n} \ldots \tau_{1}$ is then

$$
P\left(\tau_{n} \ldots \tau_{1}\right)=P_{n}\left(\frac{\lambda_{1}}{\lambda}\right)^{h}\left(\frac{\lambda_{2}}{\lambda}\right)^{l},
$$

where $h$ is the number of high priority customers, and $l$ the number of low priority customers, i.e.

$$
h=\sum_{i=1}^{n} \tau_{i}, \quad l=n-h .
$$

We can contrast the phase behaviour of the PEP with that of the EQP, where the length of the lattice is defined by the position of the last customer, and so the lattice length depends on how fast customers step into the space ahead of them (i.e. the particle hopping rate). The EQP has bounded and unbounded length phase $\$$, but with phase boundaries dependent on the hopping rate 25$]$.

\subsection{Density profiles and waiting times}

To define a density profile for the PEP we must specify both the site, $i$, and the lattice length, $n$, so that

$$
\left\langle\tau_{i}\right\rangle_{n}=P \text { (queue length is } n \text {, and site } i \text { is occupied). }
$$

These are one-point functions. We can similarly define higher order correlations

$$
\left\langle\tau_{i_{1}} \tau_{i_{2}} \ldots \tau_{i_{m}}\right\rangle_{n}, \quad n \geq i_{1}>i_{2}>\ldots>i_{m} \geq 1
$$

The rate equations for the one-point functions are

$$
\begin{aligned}
\frac{\mathrm{d}}{\mathrm{d} t}\left\langle\tau_{1}\right\rangle_{1} & =\lambda_{1} P_{0}+\mu\left\langle\tau_{2}\right\rangle_{2}-(\lambda+\mu)\left\langle\tau_{1}\right\rangle_{1}, \\
\frac{\mathrm{d}}{\mathrm{d} t}\left\langle\tau_{1}\right\rangle_{n} & =\lambda\left\langle\tau_{1}\right\rangle_{n-1}+\mu\left\langle\tau_{2}\right\rangle_{n+1}+p\left\langle\tau_{2}\left(1-\tau_{1}\right)\right\rangle_{n}-(\lambda+\mu)\left\langle\tau_{1}\right\rangle_{n}, \quad n>1, \\
\frac{\mathrm{d}}{\mathrm{d} t}\left\langle\tau_{i}\right\rangle_{i} & =\lambda_{1} P_{i-1}+\mu\left\langle\tau_{i+1}\right\rangle_{i+1}-p\left\langle\tau_{i}\left(1-\tau_{i-1}\right)\right\rangle_{i}-(\lambda+\mu)\left\langle\tau_{i}\right\rangle_{i}, \quad i>1, \\
\frac{\mathrm{d}}{\mathrm{d} t}\left\langle\tau_{i}\right\rangle_{n} & =\lambda\left\langle\tau_{i}\right\rangle_{n-1}+\mu\left\langle\tau_{i+1}\right\rangle_{n+1}+p\left\langle\tau_{i+1}\left(1-\tau_{i}\right)\right\rangle_{n} \\
-p\left\langle\tau_{i}\left(1-\tau_{i-1}\right)\right\rangle_{n}-(\lambda+\mu)\left\langle\tau_{i}\right\rangle_{n}, \quad i>1, n>i . &
\end{aligned}
$$

These couple the one-point functions to the two-point correlations, and length $n$ to length $n \pm 1$. The rate equations imply a conserved current of particles across the lattice, but because of the coupling between lengths, some care is required in how this current is defined. We will return to this for the bounded and unbounded phases separately.

$\ddagger$ convergent and divergent in their terminology 
Viewing the PEP as a queueing system, we are interested in performance measures, and how these differ for high and low priority customers. The current tells us the rate at which customers pass through the system, and from the density profile we can calculate the average waiting time for customers of each class.

To calculate waiting times, we use Little's result (see Chapter 2.1 of [32]), which states that the average waiting time, $\bar{W}_{i}$, is related to the average number of waiting customers, $\bar{N}_{i}$, for each class $i=1,2$, by

$$
\bar{N}_{i}=\lambda_{i} \bar{W}_{i}
$$

The average number of high priority customers can be calculated from the density profile as

$$
\bar{N}_{1}=\sum_{n=1}^{\infty} \sum_{i=1}^{n}\left\langle\tau_{i}\right\rangle_{n}
$$

and the average number of low priority customers is

$$
\bar{N}_{2}=\langle n\rangle-\bar{N}_{1}=\frac{\lambda}{\mu-\lambda}-\bar{N}_{1} .
$$

Here we take the total time from arrival to removal from the system as the waiting time for a customer. Our aim, now, is to compute the density profile for the PEP.

\subsection{Domain wall theory}

Domain wall theory [17,33 reduces the multi-particle dynamics of the TASEP $\S$ to the motion of a single random walker on the lattice. The TASEP boundary conditions (the particle entry and exit rates) create domains of low or high density at the boundaries. These domains extend through the lattice, and where they meet a shock, or domain wall, forms. Domain wall theory models the motion of this shock as a random walk, with the simplifying assumptions that density is constant throughout each domain, and that there is a sharp transition between domains so that the shock can be localised to a single site. Though the stationary solution of the TASEP is known exactly, domain wall theory provides a simple physical explanation of the stationary behaviour [17], and beyond this it allows accurate approximation of some dynamic properties [33. Domain wall theory can also be applied to more complex models [34,35] where the exact solution is not known. For the EQP, a domain wall approach was used to describe the global density profile in the divergent length (i.e. unbounded) phase 26. In [30 domain wall theory provided an exact solution and we will show that this also occurs in the unbounded phase of the PEP.

The PEP has a natural domain wall structure. As high priority customers overtake and reach the service end, they form a jam (Figure 2(a) : a jam is a section of high priority customers (filled sites) at the service end ahead of any low priority customer (empty site). The jam is characterised by $k$, the number of consecutive high priority customers. As there are no gaps, there is no overtaking in the jammed region, and

$\S$ Domain wall theory applies more generally to the partially asymmetric simple exclusion process. 


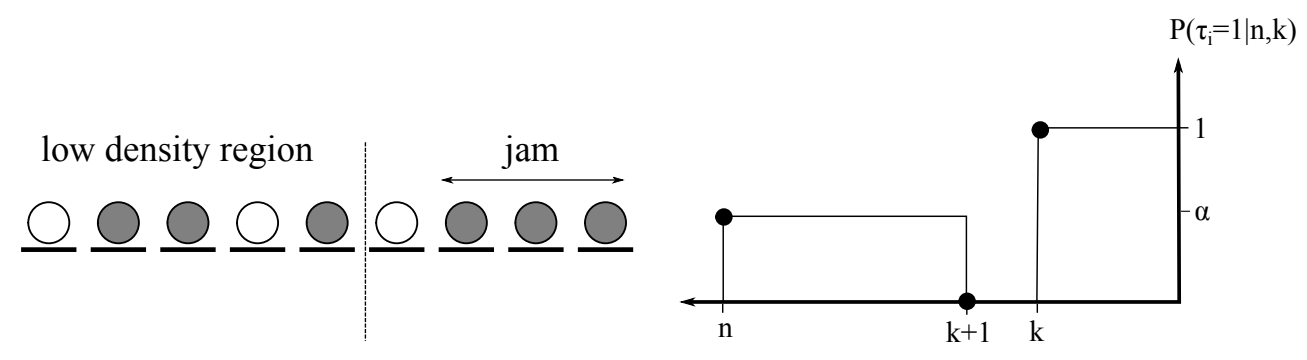

(a) A queue configuration with jam length (b) Conditional probability that site $i$ is $k=3$ filled given queue length $n$ and jam length $k$

Figure 2.

the length of the jam reduces only as customers are served. This is similar to the situation in [30], where a TASEP with parallel update and deterministic bulk motion is considered. In [36], a jam of particles was suggested as the cause of a reduced effective lattice length in the reverse bias regime of the partially asymmetric simple exclusion process.

Let us assume that the region beyond the jam has uniform density, and that the conditional probability that site $i$ is filled, given the queue length, $n$, and jam length, $k$, is (Figure 2(b)

$$
P\left(\tau_{i}=1 \mid n, k\right)= \begin{cases}1 & 1 \leq i \leq k \\ 0 & i=k+1 \\ \alpha & k+2 \leq i \leq n .\end{cases}
$$

Then the bulk equation $(n>k+1, k>0)$ for $P(n, k)$, the probability of a length $k$ jam in a length $n$ queue, is

$$
\begin{aligned}
\frac{\mathrm{d}}{\mathrm{d} t} P(n, k)= & \lambda P(n-1, k)+\mu P(n+1, k+1)+\mu(1-\alpha) \alpha^{k} P(n+1,0) \\
& +p \alpha P(n, k-1)-(\lambda+\mu+p \alpha) P(n, k) .
\end{aligned}
$$

Let us explain the meaning of each of the terms in equation (17). The term

$$
\lambda P(n-1, k)
$$

is the entry into the $(n, k)$ configuration from a length $n$ queue due to the arrival of a customer, and the terms

$$
\mu P(n+1, k+1)+\mu(1-\alpha) \alpha^{k} P(n+1,0),
$$

represent the service of a customer. The second term is the transition into the $k$-jam state from the $(k=0)$-jam state by the service of a low priority customer who was followed by $k$ consecutive high priority customers. The term

$$
p \alpha P(n, k-1),
$$

is a $(k-1)$-jam extending to length $k$ with rate $p \alpha$ : there is a high priority customer at site $k+1$ with probability $\alpha$, which overtakes with rate $p$ the low priority customer at 
site $k$ (the low priority customer marking the end of the $(k-1)$-jam). The low priority customer thus moves to position $k+1$ defining the new end of the jam. The loss terms

$$
-(\lambda+\mu+p \alpha) P(n, k)
$$

are the rate at which the $(n, k)$ configuration is left due to a customer arrival or service, or growth of the jam.

In the next section, we will show that the $n \rightarrow \infty$ limit of (17), and the corresponding equation for $k=0$, follows from the unbounded phase master equation. We find the exact stationary solutions of these equations, describing the behaviour of the jam on an infinite lattice. In the bounded phase (Section 3), domain wall theory leads to two complementary approximations. One reveals information about the length dependence and the other about waiting times.

\section{The unbounded phase}

We consider first the unbounded phase of the PEP, where the total arrival rate exceeds the service rate $(\lambda>\mu)$. Recall that the expected lattice length grows as $\langle n\rangle \sim(\lambda-\mu) t$. In our domain wall picture, the jam increases with rate $p \alpha$ and decreases with rate $\mu$ (ignoring for the moment the $\mu(1-\alpha) \alpha^{k} P(n+1,0)$ term in (17)). If $p \alpha>\mu$, the jam will grow as $(p \alpha-\mu) t$ unless it reaches the arrival end of the queue, but if $p \alpha<\mu$, the jam length will fluctuate near 0 .

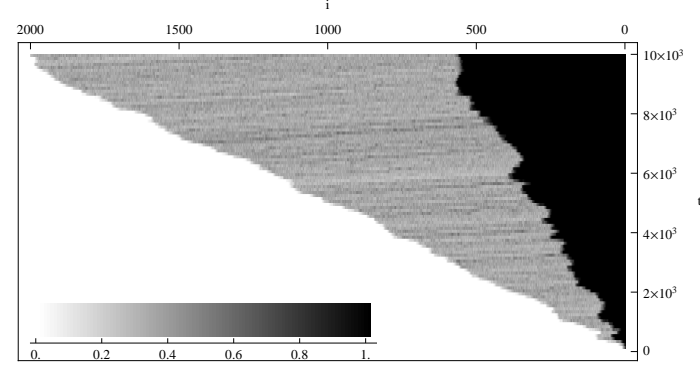

(a) With $p=3$, there is a growing jam.

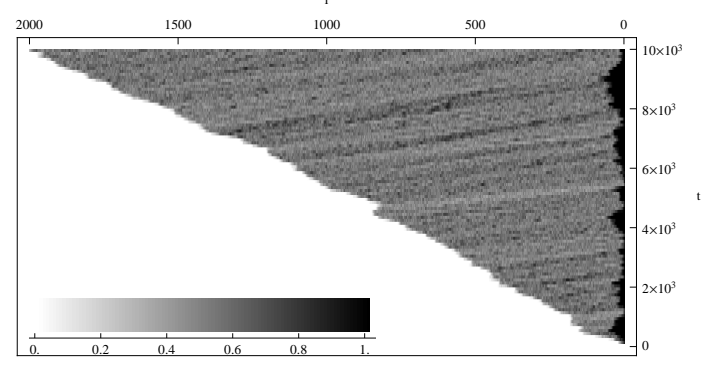

(b) With $p=1.8$ the jam remains finite.

Figure 3. Time evolution of an unbounded queue with arrival rates $\lambda_{1}=1.1$, $\lambda_{2}=0.1$, and service rate $\mu=1$. Density $\left\langle\tau_{i}\right\rangle(t)$ is averaged over a small time period, and the inset shows mapping of density to colour.

Figure 3 shows simulation results for a growing jam (Figure 3(a)], and a jam fluctuating near 0 (Figure 3(b)). The figures show the time evolution of the density profile, starting from an empty queue, with the density at each site calculated by averaging over a short time period. The queue length grows with rate $\lambda-\mu$, and the low density region beyond the jam of high priority customers is fairly regular. We will focus on the situation in Figure 3(b), where the jam length remains finite. In Figure 3(a) where the jam grows, we see that it nevertheless grows more slowly than the queue length, and we will comment on this later. 
We would like to understand the late time behaviour in the unbounded phase, but as for any fixed configuration $\tau_{n} \ldots \tau_{1}$,

$$
\lim _{t \rightarrow \infty} P\left(\tau_{n} \ldots \tau_{1}\right)=0
$$

the global description is uninformative. Instead, we follow the approach of [27] and consider local behaviour relative to a specified reference frame.

The service frame is fixed at the right hand end of the lattice where customers are served and depart. For a general but finite section of length $m$,

$$
\tau_{m} \ldots \tau_{1}
$$

the service frame probability in a length $n$ lattice is defined as

$$
P_{\text {serv }}\left(\tau_{m} \ldots \tau_{1} ; n\right)=\sum_{\tau_{n}, \ldots, \tau_{m+1}=0,1} P\left(\tau_{n} \ldots \tau_{m+1} \tau_{m} \ldots \tau_{1}\right)
$$

We will also consider the arrival frame, fixed at the left hand end of the lattice. In this case we number sites left to right. A general length $m$ section is written

$$
\tau_{1} \ldots \tau_{m}
$$

and the arrival frame probability in a length $n$ lattice is defined by

$$
P_{\text {arr }}\left(\tau_{1} \ldots \tau_{m} ; n\right)=\sum_{\tau_{m+1}, \ldots, \tau_{n}=0,1} P\left(\tau_{1} \ldots \tau_{m} \tau_{m+1} \ldots \tau_{n}\right) .
$$

In the $t \rightarrow \infty$ limit, the expected lattice length is infinite, and thus we are interested in the $n \rightarrow \infty$ limits

$$
\begin{aligned}
& P_{\text {serv }}\left(\tau_{m} \ldots \tau_{1}\right)=\lim _{n \rightarrow \infty} P_{\text {serv }}\left(\tau_{m} \ldots \tau_{1} ; n\right), \\
& P_{\text {arr }}\left(\tau_{1} \ldots \tau_{m}\right)=\lim _{n \rightarrow \infty} P_{\text {arr }}\left(\tau_{1} \ldots \tau_{m} ; n\right),
\end{aligned}
$$

with the assumption that this limit exists. We will write the rate equations for the arrival and service frame probabilities (21) in this limit, then seek the stationary solution. To do this, we use a domain wall ansatz for (21), and show that this leads to the exact stationary solution for these quantities.

\subsection{Domain wall ansatz}

We use domain wall theory to form an ansatz for the service frame probabilities. We consider a general but finite section of length $m$, with a length $k$ jam,

$$
\tau_{m} \ldots \tau_{k+2} 01^{k}=\tau 01^{k}
$$

where $1^{k}$ indicates a string of $k 1$ 's. The configuration of any finite segment can be written this way, as long as we can take $m \geq k+1$. We will assume that the conditional probability for a high at site $i$ given a jam of length $k$ is

$$
P\left(\tau_{i}=1 \mid k\right)= \begin{cases}1 & 1 \leq i \leq k \\ 0 & i=k+1 \\ \alpha & k+2 \leq i\end{cases}
$$


which is $(16)$ in the $n \rightarrow \infty$ limit. Then the probability of the finite segment $(22)$ is

$$
\begin{aligned}
P_{\text {serv }}\left(\boldsymbol{\tau} 01^{k}\right) & =\sum_{\tau_{\infty}, \ldots, \tau_{m+1}=0,1} P\left(\ldots \tau_{m+1} \tau_{m} \ldots \tau_{k+2} 01^{k}\right) \\
& =\alpha^{h}(1-\alpha)^{l} P_{\text {jam }}(k),
\end{aligned}
$$

where $h$ is the number of highs in the configuration beyond the jam up to position $m$, and $l$ is the number of lows, that is

$$
h=\sum_{i=k+2}^{m} \tau_{i}, \quad l=m-h-k-1,
$$

and $P_{\text {jam }}(k)$ is the probability of a length $k$ jam. The jam probabilities are normalised such that

$$
\sum_{k=0}^{\infty} P_{\text {jam }}(k)=1 .
$$

Equation (24) is the domain wall ansatz for the stationary service frame probabilities.

\subsection{The service frame}

In this section we write the general service frame rate equations, then apply the domain wall ansatz (24). We use the notation $\left.\boldsymbol{\tau}\right|_{(i, i-1)}$ to indicate the exchange of customers in places $i$ and $i-1$. That is, for $\boldsymbol{\tau}=\tau_{r} \ldots \tau_{1}$

$$
\left.\boldsymbol{\tau}\right|_{(i, i-1)}=\tau_{r} \ldots \tau_{i+1} \tau_{i-1} \tau_{i} \tau_{i-2} \ldots \tau_{1}
$$

and

$$
\left.0 \boldsymbol{\tau}\right|_{(r+1, r)}=\tau_{r} 0 \tau_{r-1} \ldots \tau_{1} .
$$

The stationary rate equation for the $k$-jam configuration 22 with $k \geq 1$ is

$$
\begin{aligned}
0 & =\frac{\mathrm{d}}{\mathrm{d} t} P_{\text {serv }}\left(\boldsymbol{\tau} 01^{k}\right) \\
& =\mu P_{\text {serv }}\left(\boldsymbol{\tau} 01^{k+1}\right)+\mu P_{\text {serv }}\left(\boldsymbol{\tau} 01^{k} 0\right) \\
& +p \tau_{m} P_{\text {serv }}\left(\left.0 \boldsymbol{\tau} 01^{k}\right|_{(m+1, m)}\right)+\sum_{i=k+2}^{m} p\left(1-\tau_{i}\right) \tau_{i-1} P_{\text {serv }}\left(\left.\boldsymbol{\tau} 01^{k}\right|_{(i, i-1)}\right)+p P_{\text {serv }}\left(\boldsymbol{\tau} 101^{k-1}\right) \\
& -\mu P_{\text {serv }}\left(\boldsymbol{\tau} 01^{k}\right)-\sum_{i=k+2}^{m} p \tau_{i}\left(1-\tau_{i-1}\right) P_{\text {serv }}\left(\boldsymbol{\tau} 01^{k}\right)-p\left(1-\tau_{m}\right) P_{\text {serv }}\left(1 \boldsymbol{\tau} 01^{k}\right) .
\end{aligned}
$$

Let us again explain the various terms. The terms

$$
\mu P_{\text {serv }}\left(\boldsymbol{\tau} 01^{k+1}\right)+\mu P_{\text {serv }}\left(\tau 01^{k} 0\right),
$$

give the rate of arrival to the $k$-jam configuration after, respectively, a high or low priority customer is served. Then there are the hopping terms. A high in $m$ th place in $\boldsymbol{\tau} 01^{k}$ can arrive from place $m+1$ :

$$
p \tau_{m} P_{\text {serv }}\left(\left.0 \boldsymbol{\tau} 01^{k}\right|_{(m+1, m)}\right) .
$$


Overtaking within the low density region behind the jam is given by

$$
\sum_{i=k+2}^{m} p\left(1-\tau_{i}\right) \tau_{i-1} P_{\text {serv }}\left(\left.\boldsymbol{\tau} 01^{k}\right|_{(i, i-1)}\right),
$$

and a $(k-1)$-jam extends to a $k$-jam when a high hops onto the end:

$$
p P_{\text {serv }}\left(\tau 101^{k-1}\right) \text {. }
$$

The loss term

$$
-\mu P_{\text {serv }}\left(\tau 01^{k}\right)
$$

is the reduction of the jam as a customer is served, and

$$
-\sum_{i=k+2}^{m} p \tau_{i}\left(1-\tau_{i-1}\right) P_{\text {serv }}\left(\boldsymbol{\tau} 01^{k}\right)
$$

are overtakings within the $m$ places of $\boldsymbol{\tau} 01^{k}$. The final loss term

$$
-p\left(1-\tau_{m}\right) P_{\text {serv }}\left(1 \tau 01^{k}\right)
$$

arises if the configuration has a low in $m$ th place, which can be overtaken by a high from place $m+1$. Finally we note that the terms involving the arrival rates $\lambda_{1}$ and $\lambda_{2}$ do not appear in (27) as they cancel from the stationary rate equations in the $n \rightarrow \infty$ limit.

Substituting the ansatz (24), the terms representing overtaking within the $m$ sites of $\boldsymbol{\tau} 01^{k}$ combine and telescope to

$$
\begin{aligned}
& p\left(\sum_{i=k+2}^{m}\left(1-\tau_{i}\right) \tau_{i-1}-\sum_{i=k+2}^{m} \tau_{i}\left(1-\tau_{i-1}\right)\right) \alpha^{h}(1-\alpha)^{l} P_{\mathrm{jam}}(k) \\
& =p\left(\tau_{k+1}-\tau_{m}\right) \alpha^{h}(1-\alpha)^{l} P_{\mathrm{jam}}(k) \\
& =-p \tau_{m} \alpha^{h}(1-\alpha)^{l} P_{\mathrm{jam}}(k) ;
\end{aligned}
$$

recall that $\tau_{k+1}=0$.

The factor $\alpha^{h}(1-\alpha)^{l}$ is common to all terms in the rate equation. Cancelling, and simplifying leaves

$0=p \alpha P_{\mathrm{jam}}(k-1)+\mu P_{\mathrm{jam}}(k+1)+\mu(1-\alpha) \alpha^{k} P_{\mathrm{jam}}(0)-(\mu+p \alpha) P_{\mathrm{jam}}(k)$.

This agrees with the $n \rightarrow \infty$ limit of (17), but we have derived it from the full PEP rate equations. Were it not for the $P_{\text {jam }}(0)$ term, this equation for the position of the jam would have the same form as the domain wall theory for the TASEP [33].

The $k=0$ case differs only slightly. In this case the rate equation is

$$
\begin{aligned}
0 & =\frac{\mathrm{d}}{\mathrm{d} t} P_{\text {serv }}(\boldsymbol{\tau} 0) \\
& =\mu P_{\text {serv }}(\boldsymbol{\tau} 01)+\mu P_{\text {serv }}(\boldsymbol{\tau} 00) \\
& +p \tau_{m} P_{\text {serv }}\left(\left.0 \boldsymbol{\tau} 0\right|_{(m+1, m)}\right)+\sum_{i=2}^{m} p\left(1-\tau_{i}\right) \tau_{i-1} P_{\text {serv }}\left(\left.\boldsymbol{\tau} 0\right|_{(i, i-1)}\right) \\
& -\mu P(\boldsymbol{\tau} 0)-\sum_{i=2}^{m} p \tau_{i}\left(1-\tau_{i-1}\right) P_{\text {serv }}(\boldsymbol{\tau} 0)-p\left(1-\tau_{m}\right) P_{\text {serv }}(1 \boldsymbol{\tau} 0)
\end{aligned}
$$


Substituting the ansatz (24), this reduces to

$$
0=\mu P_{\text {jam }}(1)-(\mu \alpha+p \alpha) P_{\text {jam }}(0),
$$

and rearranging gives

$$
P_{\text {jam }}(1)=\frac{p \alpha}{\mu} P_{\text {jam }}(0)+\alpha P_{\text {jam }}(0) .
$$

With this as the base case, we use (28) to show by induction that

$$
P_{\text {jam }}(k)=\frac{p \alpha}{\mu} P_{\text {jam }}(k-1)+\alpha^{k} P_{\text {jam }}(0), \quad k \geq 1 .
$$

This recurrence for $P_{\text {jam }}(k)$ has solution

$$
\begin{aligned}
P_{\text {jam }}(k) & =\sum_{i=0}^{k}\left(\frac{p \alpha}{\mu}\right)^{k-i} \alpha^{i} P_{\text {jam }}(0) \\
& =\frac{p\left(\frac{p \alpha}{\mu}\right)^{k}-\mu \alpha^{k}}{p-\mu} P_{\text {jam }}(0) .
\end{aligned}
$$

The normalisation condition (26) fixes

$$
P_{\text {jam }}(0)=(1-\alpha)\left(1-\frac{p \alpha}{\mu}\right),
$$

subject to the constraint

$$
p \alpha<\mu \text {. }
$$

The domain wall picture makes the meaning of this constraint clear. The jam of high priority customers grows with rate $p \alpha$ and is reduced with rate $\mu$. If $p \alpha>\mu$ the jam grows with rate $p \alpha-\mu>0$, that is

$$
\langle k\rangle \sim(p \alpha-\mu) t
$$

and as $t \rightarrow \infty$ the expected length of the jam becomes infinite. In contrast, when (35) is satisfied, the service rate is fast enough to prevent a backlog of high priority customers, and the expected jam length is finite and given by

$$
\langle k\rangle=\sum_{k=1}^{\infty} k P_{\mathrm{jam}}(k)=\frac{\alpha}{1-\alpha}+\frac{p \alpha}{\mu-p \alpha} .
$$

\subsection{The arrival frame}

To determine $\alpha$, we examine the PEP in the arrival frame. Sites are now numbered left to right, and the interchange operation is defined as

$$
\left.\boldsymbol{\tau}\right|_{(i, i+1)}=\tau_{1} \ldots \tau_{i-1} \tau_{i+1} \tau_{i} \tau_{i+2} \ldots \tau_{r}
$$

for $\boldsymbol{\tau}=\tau_{1} \ldots \tau_{r}$.

We will assume that the jam is always far from the arrival end. In the late time limit this is guaranteed if

$$
p \alpha<\lambda \text {. }
$$


This condition is clearly met when (35) is satisfied, but we will show that $\alpha$ can be determined consistently with this requirement. Then in the arrival frame, the ansatz (24) implies that for a configuration on the first $m$ sites,

$$
\boldsymbol{\tau}=\tau_{1} \ldots \tau_{m}
$$

the arrival frame probability has the form

$$
P_{\text {arr }}(\boldsymbol{\tau})=\alpha^{h}(1-\alpha)^{l}
$$

where

$$
h=\sum_{i=1}^{m} \tau_{i}, \quad l=m-h .
$$

The stationary rate equation for this configuration is

$$
\begin{aligned}
0 & =\frac{\mathrm{d}}{\mathrm{d} t} P_{\mathrm{arr}}(\boldsymbol{\tau}) \\
& =\lambda_{1} \tau_{1} P_{\mathrm{arr}}\left(\tau_{2} \ldots \tau_{m}\right)+\lambda_{2}\left(1-\tau_{1}\right) P_{\mathrm{arr}}\left(\tau_{2} \ldots \tau_{m}\right) \\
& +\sum_{i=1}^{m-1} p\left(1-\tau_{i}\right) \tau_{i+1} P_{\mathrm{arr}}\left(\left.\boldsymbol{\tau}\right|_{(i, i+1)}\right)+p\left(1-\tau_{m}\right) P_{\mathrm{arr}}\left(\left.\boldsymbol{\tau} 1\right|_{(m, m+1)}\right) \\
& -\lambda P_{\mathrm{arr}}(\boldsymbol{\tau})-\sum_{i=1}^{m-1} p \tau_{i}\left(1-\tau_{i+1}\right) P_{\mathrm{arr}}(\boldsymbol{\tau})-p \tau_{m} P_{\mathrm{arr}}(\boldsymbol{\tau} 0) .
\end{aligned}
$$

Substituting (39), the summed hopping terms again combine and telescope, and the factors of $\alpha$ and $1-\alpha$ common to all terms can be cancelled. This leaves

$$
0=-\lambda \alpha(1-\alpha)+p \alpha^{2}(1-\alpha)-p \tau_{1} \alpha(1-\alpha)+\tau_{1} \lambda_{1}(1-\alpha)+\left(1-\tau_{1}\right) \lambda_{2} \alpha,
$$

which for both $\tau_{1}=0$ and $\tau_{1}=1$ reduces to

$$
p \alpha^{2}-(p+\lambda) \alpha+\lambda_{1}=0
$$

The two solutions are

$$
\alpha_{ \pm}=\frac{p+\lambda \pm \sqrt{(p-\lambda)^{2}+4 p \lambda_{2}}}{2 p}
$$

with $0<\alpha_{-}<1$ and $\alpha_{+}>1$ for $p, \lambda_{1}, \lambda_{2}>0$. As $\alpha$ is a density value we must take $\alpha=\alpha_{-}$. Substituting this value for $\alpha$, it is seen that $p \alpha<\lambda$ (the constraint (38)) is satisfied for all physical parameter values. The jam always grows more slowly than the queue length, as illustrated by the simulation results in Figure $3(\mathrm{a})$. In the limit $p \rightarrow 0$, $\alpha \rightarrow \lambda_{1} / \lambda$, the expected occupancy of any site when there is no overtaking.

With $\alpha$ given by (41), and the jam probabilities, $P_{\text {jam }}(k)$ by (33) and (34), we thus have the stationary domain wall solution describing the motion of the high priority jam on an infinite lattice. Through (24) and (39), this then gives the exact stationary service and arrival frame probabilities $P_{\text {serv }}\left(\tau_{m} \ldots \tau_{1}\right)$ and $P_{\text {arr }}\left(\tau_{1} \ldots \tau_{m}\right)$. In the next section, we compare this solution to simulation results, where the lattice length, $n$, is large, but necessarily finite. In doing so we make the assumption that the large $n$ behaviour converges to the $n \rightarrow \infty$ limit.

We note also that the result (34), the probability to not have a jam, can be understood by the following heuristic argument $\mid$. The number of low priority customers $\|$ We thank an anonymous referee for pointing this out. 

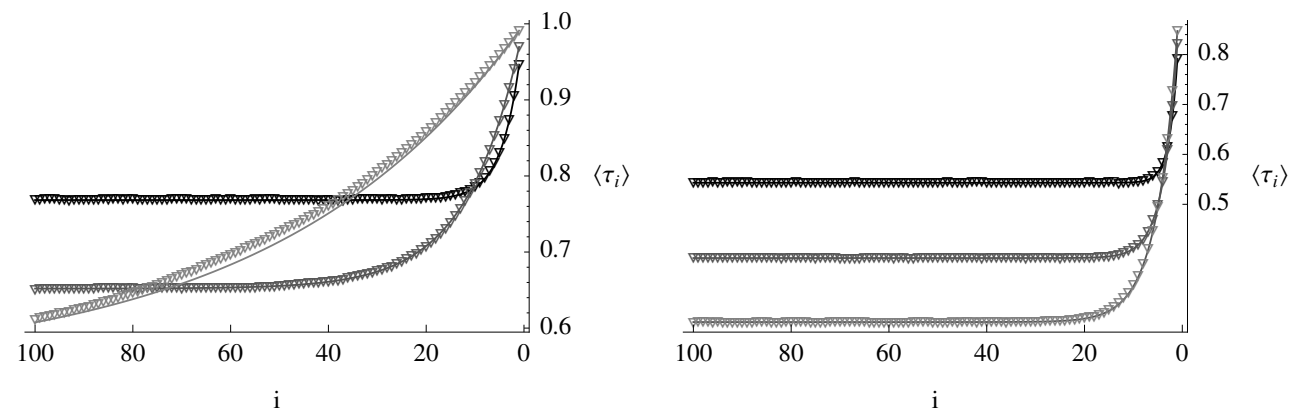

(a) Density profile at the service end of the (b) Density profile at the service end of the unbounded queue; $\lambda_{1}=1.1, \lambda_{2}=0.1, \mu=$ unbounded queue; $\lambda_{1}=0.9, \lambda_{2}=0.3, \mu=$ 1 with $p=1$ (black), $p=1.4$ (mid-gray), 1 with $p=1$ (black), $p=1.8$ (mid-gray), and $p=1.8$ (light gray). and $p=3$ (light gray).

Figure 4. Density profiles of the unbounded PEP in the service frame. Triangle markers for simulation results plotted against calculated profile $\left\langle\tau_{i}\right\rangle$.

in the system at time $t$ can be expressed as $(1-\alpha)(\lambda-\mu) t$, i.e. the ratio of low priority customers $(1-\alpha)$ times the expected length of the queue. It can also be expressed as $\left(\lambda_{2}-P_{\text {jam }}(0) \mu\right) t$, i.e. (arrival rate - service rate) times time. Comparing the two expressions and using (41) we obtain (34).

\subsection{Density profile, conserved currents, and service rates}

The density at site $i$ in the service frame, $\left\langle\tau_{i}\right\rangle_{\infty}$, is computed from the domain wall solution as

$$
\begin{aligned}
\left\langle\tau_{i}\right\rangle_{\infty} & =\alpha \sum_{k=0}^{i-2} P_{\mathrm{jam}}(k)+\sum_{k=i}^{\infty} P_{\mathrm{jam}}(k) \\
& =\alpha+(1-\alpha)\left(\frac{p \alpha}{\mu}\right)^{i} .
\end{aligned}
$$

Figure 4 shows $\left\langle\tau_{i}\right\rangle_{\infty}$ computed from (42) plotted against simulation results for a range of parameters chosen, necessarily, with $p \alpha<\mu$. In all cases we see excellent agreement. The only deviation occurs for $p=1.8$ in Figure 4(a), which with $p \alpha \simeq 0.98$ is very close to the critical value $p \alpha=\mu$. We believe that this difference occurs because of the slowing convergence of simulation results as we near the critical point. A study of the critical behaviour, as for example the numerical study [29] for the EQP, would be required to confirm this. With $p \alpha>\mu$, the jam grows to fill any finite section at the service end. Checking Figure 3(a), we see that the rate of growth is consistent with $\langle k\rangle \sim(p \alpha-\mu) t$.

We can check that the domain wall solution satisfies the rate equations for the one point functions $(9)-(12)$. To obtain the service frame rate equations, we take the $n \rightarrow \infty$ limit, allowing us to neglect the boundary cases. The bulk equations can be 
written

where

$$
\frac{\mathrm{d}}{\mathrm{d} t}\left\langle\tau_{i}\right\rangle_{\infty}=J_{\infty}^{(i+1)}-J_{\infty}^{(i)}, \quad i \geq 1
$$

$$
\begin{aligned}
& J_{\infty}^{(1)}=\mu\left\langle\tau_{1}\right\rangle_{\infty} \\
& J_{\infty}^{(i)}=\mu\left\langle\tau_{i}\right\rangle_{\infty}+p\left\langle\tau_{i}\left(1-\tau_{i-1}\right)\right\rangle_{\infty}, \quad i \geq 2 .
\end{aligned}
$$

In the stationary state the time derivatives are zero so 43 defines a conserved current

$$
J_{\infty}=J_{\infty}^{(1)}=J_{\infty}^{(2)}=\ldots
$$

The bulk current, $J_{\infty}^{(i)}$, has the usual TASEP hopping term, $p\left\langle\tau_{i}\left(1-\tau_{i-1}\right)\right\rangle_{\infty}$. The additional term $\mu\left\langle\tau_{i}\right\rangle_{\infty}$ arises due to the choice of reference frame.

The two-point correlation $\left\langle\tau_{i+1}\left(1-\tau_{i}\right)\right\rangle_{\infty}$ is computed as

$$
\begin{aligned}
\left\langle\tau_{i+1}\left(1-\tau_{i}\right)\right\rangle_{\infty} & =\alpha(1-\alpha) \sum_{k=0}^{i-2} P_{\mathrm{jam}}(k)+\alpha P_{\mathrm{jam}}(i-1) \\
& =\alpha(1-\alpha)\left(1-\left(\frac{p \alpha}{\mu}\right)^{i}\right) .
\end{aligned}
$$

The resulting service frame current is

$$
J_{\infty}=p \alpha(1-\alpha)+\mu \alpha
$$

and is the rate at which particles exit the system.

In terms of the queueing model, the current is the average rate at which high priority customers leave the queue. As the total rate at which customers leave the system is $\mu$. low priority customers leave the queue at rate

$$
\mu-J_{\infty}=(\mu-p \alpha)(1-\alpha) .
$$

The constraint $p \alpha<\mu$ (equation (35)) ensures that this rate is greater than zero and low priority customers always receive a share of the service. For $p \alpha>\mu$, the jam of high priority customers at the service end becomes unbounded, and low priority customers can no longer reach the front of the queue to be served. Thus the low priority current has a second order phase transition at $p \alpha=\mu$.

The phase transition subdivides the unbounded phase of the PEP. By fixing values for $\lambda=\lambda_{1}+\lambda_{2}$ and $\mu$, we can plot illustrative two dimensional phase diagrams with $\lambda_{1}$ and $p$ as the axes. Using (41) for $\alpha=\alpha_{-}$, the curve where $p \alpha=\mu$ is given by

$$
\lambda_{1}^{(\infty)}(p)= \begin{cases}\lambda & p<\mu \\ \mu\left(1+\frac{\lambda-\mu}{p}\right) & p \geq \mu,\end{cases}
$$

and $p \alpha<\mu$ for $\lambda_{1}<\lambda_{1}^{(\infty)}(p)$. Figure 5 shows the phase diagram for $\lambda=1.2, \mu=1$. The function $\lambda_{1}^{(\infty)}(p)$ is decreasing in $p$ and $\lim _{p \rightarrow \infty} \lambda_{1}(p)=\mu$. Therefore, the transition into the 'infinite jam' phase occurs only if $\lambda_{1}>\mu$. This is marked by the lower dashed line. The upper dashed line marks the $\lambda_{1}=\lambda, \lambda_{2}=0$ boundary.

I If the queue was ever empty, the rate at which customers leave would be less than the service rate, but in the unbounded phase this is not a concern. 


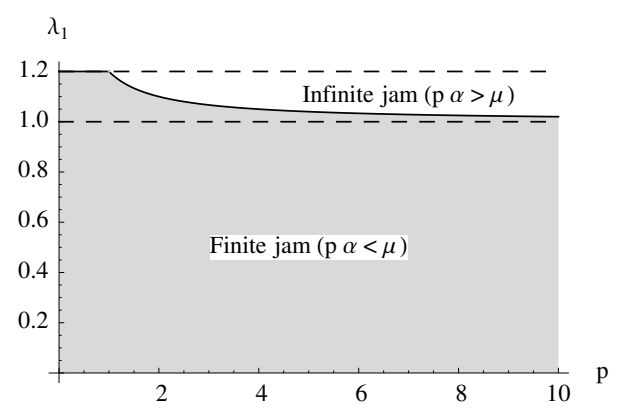

Figure 5. Subdivisions of the unbounded phase with $\lambda=1.2, \mu=1$.

\section{The bounded phase}

In the queueing theory interpretation, it is the bounded phase of the PEP (with $\lambda<\mu$ ) that is of greatest interest. In this phase the queue lengths and waiting times remain finite, and we can compare how the waiting time varies with the overtake rate, $p$, for high and low priority class customers.

The fluctuating lattice length proves a challenge in applying domain wall theory directly to the bounded phase. We will take two approaches, each leading to an approximate solution revealing different aspects of the system. The first method tells us about the shape and length dependence of the density profiles, while the second method allows us to calculate customer waiting times.

\subsection{Domain wall ansatz}

To apply the domain wall ansatz directly in the bounded phase, we consider a general length $n$ configuration with a length $k$ jam,

$$
\tau_{n} \ldots \tau_{k+2} 01^{k}=\boldsymbol{\tau} 01^{k} .
$$

The stationary rate equation for $k>0, n>k+1$ is

$$
\begin{aligned}
0 & =\frac{\mathrm{d}}{\mathrm{d} t} P\left(\boldsymbol{\tau} 01^{k}\right) \\
& =\lambda_{1} \tau_{n} P\left(\tau_{n-1} \ldots \tau_{k+2} 01^{k}\right)+\lambda_{2}\left(1-\tau_{n}\right) P\left(\tau_{n-1} \ldots \tau_{k+2} 01^{k}\right) \\
& +\sum_{i=k+2}^{n} p\left(1-\tau_{i}\right) \tau_{i-1} P\left(\left.\boldsymbol{\tau} 01^{k}\right|_{(i, i-1)}\right)+p P\left(\boldsymbol{\tau} 101^{k-1}\right)+\mu P\left(\boldsymbol{\tau} 01^{k+1}\right)+\mu P\left(\boldsymbol{\tau} 01^{k} 0\right) \\
& -(\lambda+\mu) P\left(\boldsymbol{\tau} 01^{k}\right)-\sum_{i=k+2}^{n} p \tau_{i}\left(1-\tau_{i-1}\right) P\left(\boldsymbol{\tau} 01^{k}\right),
\end{aligned}
$$

and for $k=0, n>1$

$$
\begin{aligned}
0 & =\frac{\mathrm{d}}{\mathrm{d} t} P(\boldsymbol{\tau} 0) \\
& =\lambda_{1} \tau_{n} P\left(\tau_{n-1} \ldots \tau_{2} 0\right)+\lambda_{2}\left(1-\tau_{n}\right) P\left(\tau_{n-1} \ldots \tau_{2} 0\right) \\
& +\sum_{i=2}^{n} p\left(1-\tau_{i}\right) \tau_{i-1} P\left(\left.\boldsymbol{\tau} 0\right|_{(i, i-1)}\right)+\mu P(\boldsymbol{\tau} 01)+\mu P(\boldsymbol{\tau} 00)
\end{aligned}
$$




$$
-(\lambda+\mu) P(\boldsymbol{\tau} 0)-\sum_{i=2}^{n} p \tau_{i}\left(1-\tau_{i-1}\right) P(\boldsymbol{\tau} 0) .
$$

These are "bulk" equations, valid when the jam is away from the arrival end of the queue and are of the form discussed in Section 1.4. To see this, define

$$
P(n, k)=\sum_{\tau_{n}, \ldots, \tau_{k+2}=0,1} P\left(\tau_{n} \ldots \tau_{k+2} 01^{k}\right),
$$

which is the probability of a length $k$ jam in a length $n$ queue. For $k>0, n>k+1$, summing (51) and applying the domain wall ansatz (16) gives

$$
\begin{aligned}
0=\frac{\mathrm{d}}{\mathrm{d} t} P(n, k)= & \lambda P(n-1, k)+\mu P(n+1, k+1)+\mu(1-\alpha) \alpha^{k} P(n+1,0) \\
& +p \alpha P(n, k-1)-(\lambda+\mu+p \alpha) P(n, k) .
\end{aligned}
$$

which is exactly (17). And for $k=0, n>1$, summing 52 gives

$$
\begin{aligned}
0 & =\frac{\mathrm{d}}{\mathrm{d} t} P(n, 0) \\
& =\lambda P(n-1,0)+\mu P(n+1,1)+\mu(1-\alpha) P(n+1,0)-(\lambda+\mu+p \alpha) P(n, 0) .
\end{aligned}
$$

The simple domain wall picture breaks down when the jam reaches the arrival end, i.e. for configurations $01^{n-1}$ or $1^{n}$. Our strategy is to find a solution of the bulk equations, without requiring it to satisfy these boundary equations. We can hope that this will give an approximation to the true solution. What we will show is that, within the range of validity, the approximation is very good.

3.1.1. Length assumption. To solve the bulk equations we assume the length dependence factorises as

$$
P(n, k)=P_{n} P_{\text {jam }}^{*}(k),
$$

where $P_{n}$ is the length distribution (3). Then equation (54), for $k>0$, becomes

$$
0=p \alpha P_{\text {jam }}^{*}(k-1)+\lambda P_{\text {jam }}^{*}(k+1)+\lambda(1-\alpha) \alpha^{k} P_{\text {jam }}^{*}(0)-(\lambda+p \alpha) P_{\text {jam }}^{*}(k),
$$

and equation (55), for $k=0$, gives

$$
0=\lambda P_{\text {jam }}^{*}(1)-(\lambda \alpha+p \alpha) P_{\text {jam }}^{*}(0) .
$$

These have the same form as the unbounded queue domain wall equations, (28), (30), but with $\lambda$ in place of $\mu$. Therefore they are solved by

$$
\begin{aligned}
P_{\mathrm{jam}}^{*}(k) & =\sum_{i=0}^{k}\left(\frac{p \alpha}{\lambda}\right)^{k-i} \alpha^{i} P_{\mathrm{jam}}^{*}(0) \\
& =\frac{p\left(\frac{p \alpha}{\lambda}\right)^{k}-\lambda \alpha^{k}}{p-\lambda} P_{\mathrm{jam}}^{*}(0) .
\end{aligned}
$$

The normalisation of $P_{\text {jam }}^{*}(k)$ must be independent of $n$. For the solution to be valid for $n \rightarrow \infty$ (as there is no cap on queue length) we must require

$$
\sum_{k=0}^{\infty} P_{\text {jam }}^{*}(k)=1
$$


fixing

$$
P_{\mathrm{jam}}^{*}(0)=(1-\alpha)\left(1-\frac{p \alpha}{\lambda}\right)
$$

subject to the constraint

$$
p \alpha<\lambda \text {. }
$$

The total probability at each length, $n$, must sum to the length distribution (3), that is

$$
\sum_{k=0}^{n-1} P(n, k)+P\left(1^{n}\right)=P_{n} .
$$

As only $P\left(1^{n}\right)$ is undetermined, we must have that

$$
\begin{aligned}
P\left(1^{n}\right) & =P_{n}-\sum_{k=0}^{n-1} P(n, k) \\
& =P_{n} \sum_{k=n}^{\infty} P_{\mathrm{jam}}^{*}(k) \\
& =P_{n} \frac{p(1-\alpha)\left(\frac{p \alpha}{\lambda}\right)^{n}-\lambda\left(1-\frac{p \alpha}{\lambda}\right) \alpha^{n}}{p-\lambda} .
\end{aligned}
$$

This is analogous to the unbounded queue. There the probability that the first $n$ sites from the service end are filled is $\sum_{k=n}^{\infty} P_{\text {jam }}(k)$.

To determine $\alpha$ we return to the general $k$-jam equation $\sqrt{51+}$, and apply the domain wall ansatz (16) and the length assumption (56), leaving

$$
\begin{aligned}
0= & \frac{\lambda_{1} \mu}{\lambda} \tau_{n}(1-\alpha) P_{\text {jam }}^{*}(k)+\frac{\lambda_{2} \mu}{\lambda}\left(1-\tau_{n}\right) \alpha P_{\text {jam }}^{*}(k)+p \alpha^{2}(1-\alpha) P_{\text {jam }}^{*}(k-1) \\
& +\lambda \alpha(1-\alpha) P_{\text {jam }}^{*}(k+1)+\lambda(1-\alpha)^{2} \alpha^{k+1} P_{\text {jam }}^{*}(0) \\
& -\left(\lambda+\mu+p \tau_{n}\right) \alpha(1-\alpha) P_{\text {jam }}^{*}(k) .
\end{aligned}
$$

Multiplying (57) by $\alpha(1-\alpha)$ and subtracting from (65), we then consider $\tau_{n}=0$ and $\tau_{n}=1$ separately. Both cases reduce to

$$
0=p \alpha^{2}-(p+\mu) \alpha+\frac{\lambda_{1} \mu}{\lambda}
$$

with solutions

$$
\alpha_{ \pm}=\frac{p+\mu \pm \sqrt{(p+\mu)^{2}-4 p \frac{\lambda_{1} \mu}{\lambda}}}{2 p} .
$$

Using the inequalities

$$
\lambda_{1}<\frac{\lambda_{1} \mu}{\lambda}<\mu
$$

(the first inequality holds as the queue is bounded) we see that $0<\alpha_{-}<1$, and $\alpha_{+}>1$ when $p, \lambda_{1}, \lambda_{2}, \mu>0$. Again, we must take $\alpha=\alpha_{-}$to have a proper density value. As for the unbounded queue, in the limit $p \rightarrow 0, \alpha \rightarrow \lambda_{1} / \lambda$, the expected occupancy of any site when there is no overtaking.

+ This is for $k>0$, but $\sqrt{52}$ for $k=0$ gives the same result. 
3.1.2. Density profile. To summarise, we have solved the bulk equations in the domain wall approximation, giving the solution in the form (56), which with (3) and (59) results in

$$
P(n, k)=\left(1-\frac{\lambda}{\mu}\right)\left(1-\frac{p \alpha}{\lambda}\right) \frac{1-\alpha}{p-\lambda}\left(\frac{\lambda}{\mu}\right)^{n}\left(p\left(\frac{p \alpha}{\lambda}\right)^{k}-\lambda \alpha^{k}\right) .
$$

In general this solution does not satisfy the boundary equations for $k=n, n-1$, i.e. the cases we neglected were where the jam extends to the length of the queue. The jam grows with rate $p \alpha$, so the constraint $p \alpha<\lambda$ (equation (62) arising from the normalisation condition) requires that the queue length grows faster on average then the jam. Since we have totally neglected the boundary equations, we expect our approximation to be best when $p \alpha \ll \lambda$. In fact when $p \rightarrow 0(69)$ reduces to

$$
\lim _{p \rightarrow 0} P(n, k)=\left(1-\frac{\lambda}{\mu}\right)\left(\frac{\lambda}{\mu}\right)^{n} \frac{\lambda_{2} \lambda_{1}^{k}}{\lambda^{k+1}},
$$

as expected from the exact solution in this case, (6).

The density at site $i$ in a length $n$ queue, computed from (69), (64) is

$$
\begin{aligned}
\left\langle\tau_{i}\right\rangle_{n} & =\alpha \sum_{k=0}^{i-2} P(n, k)+\sum_{k=i}^{n-1} P(n, k)+P\left(1^{n}\right) \\
& =\alpha \sum_{k=0}^{i-2} P(n, k)+\sum_{k=i}^{\infty} P(n, k) \\
& =P_{n}\left(\alpha+(1-\alpha)\left(\frac{p \alpha}{\lambda}\right)^{i}\right) .
\end{aligned}
$$

Figure 6 shows length dependent density profiles for $p \alpha<\lambda$ (Figure 6(a)) and $p \alpha>\lambda$ (Figure 6(b)), scaled by dividing out the length distribution $P_{n}$. Triangle markers show

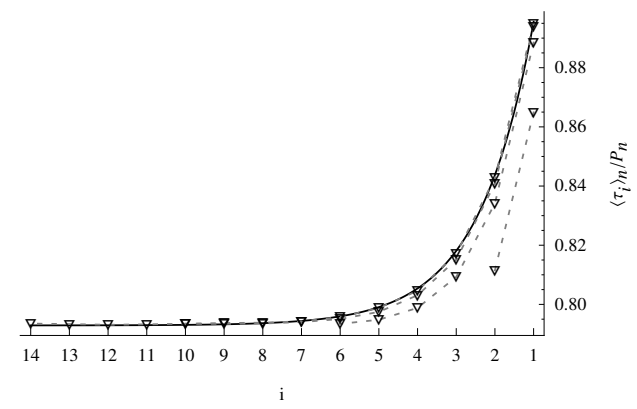

(a) $\lambda_{1}=0.7, \lambda_{2}=0.1, \mu=1, p=0.5$, giving $p \alpha<\lambda$.

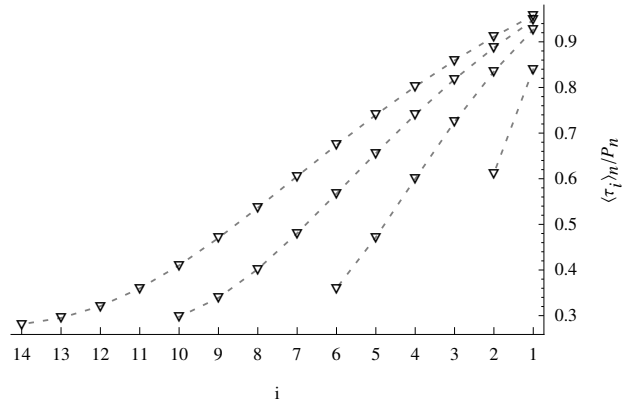

(b) $\lambda_{1}=0.7, \lambda_{2}=0.1, \mu=1, p=3$, giving $p \alpha>\lambda$.

Figure 6. Scaled density profiles $\left\langle\tau_{i}\right\rangle_{n} / P_{n}$ for $n=2,4,6,10,14$. Triangle markers for simulation results with points for each length $n$ connected by dashed lines. The analytical expression, calculated from (71), is plotted as the solid curve in (a).

simulation results, with points for each length connected by dashed lines.

In Figure 6(a), the solid curve shows $\left\langle\tau_{i}\right\rangle_{n} / P_{n}$ calculated from (71) and (3). We see that as $n$ increases, the simulation results converge to the calculated profile, and even 
for $n=10$ the match is very good. In Figure 6(b), where the solution (71) no longer applies, the profiles become almost linear and are reminiscent of the behaviour of the TASEP on the coexistence line. In that phase, the domain wall occurs at all positions with equal probability, resulting, on average, in a linear profile [12]. In the bounded PEP the evidence suggests that the constraint $p \alpha<\lambda$ marks a crossover between the localised jam, for $p \alpha<\lambda$ and the delocalised jam for $p \alpha>\lambda$.

\subsection{Aggregate density profile and current}

In this section we take a different approach, working with the rate equations for the one-point functions. We start by defining a conserved current for the bounded phase. To do so, we sum the density at each position over all lengths, thus aggregating the effect of the length fluctuations. Define the summed one-point functions

$$
\overline{\left\langle\tau_{i}\right\rangle}=\sum_{n=i}^{\infty}\left\langle\tau_{i}\right\rangle_{n}
$$

Note that as $\left\langle\tau_{i}\right\rangle_{n} \leq P_{n}$,

$$
\overline{\left\langle\tau_{i}\right\rangle} \leq \sum_{n=i}^{\infty} P_{n}=\left(\frac{\lambda}{\mu}\right)^{i},
$$

so the sum is bounded, and it converges as $\sum_{n=i}^{M}\left\langle\tau_{i}\right\rangle_{n}$ is monotone increasing in $M$. Thus $\overline{\left\langle\tau_{i}\right\rangle}$ is well defined. Higher order summed correlations are defined similarly, e.g.

$$
\overline{\left\langle\tau_{i+1}\left(1-\tau_{i}\right)\right\rangle}=\sum_{n=i}^{\infty}\left\langle\tau_{i+1}\left(1-\tau_{i}\right)\right\rangle_{n}
$$

Summing the rate equations $(9)-(12)$ gives

$\frac{\mathrm{d}}{\mathrm{d} t} \overline{\left\langle\tau_{1}\right\rangle}=\lambda_{1} P_{0}+\mu \overline{\left\langle\tau_{2}\right\rangle}+p \overline{\left\langle\tau_{2}\left(1-\tau_{1}\right)\right\rangle}-\mu \overline{\left\langle\tau_{1}\right\rangle}$,

$\frac{\mathrm{d}}{\mathrm{d} t} \overline{\left\langle\tau_{i}\right\rangle}=\lambda_{1} P_{i-1}+\mu \overline{\left\langle\tau_{i+1}\right\rangle}+p \overline{\left\langle\tau_{i+1}\left(1-\tau_{i}\right)\right\rangle}-p \overline{\left\langle\tau_{i}\left(1-\tau_{i-1}\right\rangle\right.}-\mu \overline{\left\langle\tau_{i}\right\rangle}, \quad i>1$.

These can be written as a conservation equation for three currents

$$
\frac{\mathrm{d}}{\mathrm{d} t} \overline{\left\langle\tau_{i}\right\rangle}=J_{\mathrm{ext}}^{(i)}+\bar{J}^{(i+1, i)}-\bar{J}^{(i, i-1)}, \quad i \geq 1,
$$

where

$$
\begin{aligned}
& J_{\text {ext }}^{(i)}=\lambda_{1} P_{i-1} \\
& \bar{J}^{(1,0)}=\mu \overline{\left\langle\tau_{1}\right\rangle} \\
& \bar{J}^{(i, i-1)}=\mu \overline{\left\langle\tau_{i}\right\rangle}+p \overline{\left\langle\tau_{i}\left(1-\tau_{i-1}\right)\right\rangle}, \quad i \geq 2 .
\end{aligned}
$$

$\bar{J}^{(i, i-1)}$ is the site-to-site current with a hopping term and frame current term. But customers can also step directly into place at the end of the queue, which gives the external current $J_{\text {ext }}^{(i)}$.

In the stationary distribution the time derivatives are zero, and so $(77)$ gives a recurrence for the site-to-site current

$$
\bar{J}^{(i+1, i)}=\bar{J}^{(i, i-1)}-J_{\mathrm{ext}}^{(i)},
$$


which reduces to

$$
\bar{J}^{(i+1, i)}=\bar{J}^{(1,0)}-\lambda_{1} \sum_{n=0}^{i-1} P_{n} .
$$

We have $\lim _{i \rightarrow \infty} \bar{J}^{(i+1, i)}=0$, as both terms on the right hand side of 80 go to zero. Therefore 82 implies that

$$
\bar{J}^{(1,0)}=\lambda_{1} \sum_{n=0}^{\infty} P_{n}=\lambda_{1}
$$

and

$$
\bar{J}^{(i, i-1)}=\lambda_{1}\left(1-\sum_{n=0}^{i-2} P_{n}\right)=\lambda_{1}\left(\frac{\lambda}{\mu}\right)^{i-1}, \quad i \geq 1 .
$$

Now (79) tells us that

$$
\overline{\left\langle\tau_{1}\right\rangle}=\frac{\lambda_{1}}{\mu} .
$$

This result is exact - it is the probability that the lattice is at least length one with a particle in site 1.

We could have derived this result directly from the notion of the PEP as a queue: high priority customers leave the system at an average rate $\mu \overline{\left\langle\tau_{1}\right\rangle}$. The rate at which high priority customers leave the system cannot be higher than $\lambda_{1}$, the rate they arrive. But as the service capacity exceeds the total arrival rate $\left(\lambda=\lambda_{1}+\lambda_{2}\right)$ there is no bottleneck at the server, so high priority customers* leave at the rate they arrive, that is $\mu \overline{\left\langle\tau_{1}\right\rangle}=\lambda_{1}$.

The presence of two-point correlations prevent us from calculating exact densities for $i=2,3$, etc. The mean field method [11,27] is the standard way to deal with this, assuming the correlation between neighbouring sites is small so that the two-point correlations can be approximated as products of the one-point functions. But for the PEP, there is a similarity to the unbounded system, which we can exploit to solve the one-point rate equations.

By the definition $\overline{72}, \overline{\left\langle\tau_{i}\right\rangle}$ is the probability

$$
\overline{\left\langle\tau_{i}\right\rangle}=P\left(\tau_{i}=1 \text {, length } n \geq i\right) .
$$

We can instead work with the conditional probability

$$
\left\langle\tau_{i} \mid n \geq i\right\rangle=P\left(\tau_{i}=1 \mid \text { length } n \geq i\right) ;
$$

the two are related by

$$
\overline{\left\langle\tau_{i}\right\rangle}=P(\text { length } n \geq i)\left\langle\tau_{i} \mid n \geq i\right\rangle=\left(\frac{\lambda}{\mu}\right)^{i}\left\langle\tau_{i} \mid n \geq i\right\rangle .
$$

Similarly, define $\left\langle\tau_{i}\left(1-\tau_{i-1}\right) \mid n \geq i\right\rangle$ through

$$
\overline{\left\langle\tau_{i}\left(1-\tau_{i-1}\right)\right\rangle}=\left(\frac{\lambda}{\mu}\right)^{i}\left\langle\tau_{i}\left(1-\tau_{i}\right) \mid n \geq i\right\rangle .
$$

\footnotetext{
* A corresponding argument applies to low priority customers.
} 
Substituting into 79, , 80 ) gives

$$
\begin{aligned}
\frac{\lambda_{1} \mu}{\lambda} & =\mu\left\langle\tau_{1} \mid n \geq 1\right\rangle \\
& =\mu\left\langle\tau_{i} \mid n \geq i\right\rangle+p\left\langle\tau_{i}\left(1-\tau_{i-1}\right) \mid n \geq i\right\rangle, \quad i \geq 2 .
\end{aligned}
$$

These have the same form as the current equations for the unbounded queue, (44), with an effective current

$$
\widetilde{J}=\frac{\lambda_{1} \mu}{\lambda}
$$

so are solved by the unbounded queue one- and two-point functions (42), 46). That is

$$
\left\langle\tau_{i} \mid n \geq i\right\rangle=\alpha+(1-\alpha)\left(\frac{p \alpha}{\mu}\right)^{i}, \quad i \geq 1
$$

and

$$
\left\langle\tau_{i}\left(1-\tau_{i-1}\right) \mid n \geq i\right\rangle=\alpha(1-\alpha)\left(1-\left(\frac{p \alpha}{\mu}\right)^{i-1}\right), \quad i \geq 2 .
$$

To determine $\alpha$, we substitute (92), (93) into (90), and take $i \rightarrow \infty$ (assuming $p \alpha<\mu$ ). This gives back the quadratic for $\alpha(66)$, so we again must take $\alpha=\alpha_{-}$, given by (67). Note that $p \alpha<\mu$ if $p, \mu, \lambda_{2}>0$, so the density profile is always exponentially decaying.

This solution satisfies the current equation (90), and therefore the resulting aggregated one- and two-point functions (88), (89) satisfy the one-point rate equations (75), (76). But as it does not in general satisfy the higher order rate equations it is approximate only.

Figure 7 compares simulated and calculated aggregated density profiles, $\overline{\left\langle\tau_{i}\right\rangle}$. We have divided out the length dependence factor $(\lambda / \mu)^{i}$ so in fact are plotting $\left\langle\tau_{i} \mid n \geq i\right\rangle$ computed from (92). We see that at $i=1$, where the exact value $\left\langle\tau_{1} \mid n \geq 1\right\rangle$ is known, and asymptotically for large $i$, the simulated and calculated density profiles agree. At intermediate values of $i$ we see the greatest discrepancy, indicating that the aggregated domain wall solution is approximate only, although the agreement is still very good.

Note we could also compare the aggregated profiles with $\left\langle\tau_{i}\right\rangle_{n}$ (71) summed over $n$. However, even at position 1 the summed $\left\langle\tau_{1}\right\rangle_{n}$ does not agree with $\overline{\left\langle\tau_{1}\right\rangle} 85$ for which the exact result is known. The direct application of the domain wall ansatz in Section 3.1 gave an indication of the length dependence in the system, but the approach in this section, following from the current conservation equation, is in much better agreement numerically with simulation results.

\subsection{Waiting times}

We can use the aggregated one-point functions to compute the average number of customers in the queue, and in turn the average waiting times for both classes of customers. Switching the order of the sums in $(14)$, we can write $\bar{N}_{1}$, the average number of high priority customers, as

$$
\bar{N}_{1}=\sum_{i=1}^{\infty} \sum_{n=i}^{\infty}\left\langle\tau_{i}\right\rangle_{n}=\sum_{i=1}^{\infty} \overline{\left\langle\tau_{i}\right\rangle} .
$$




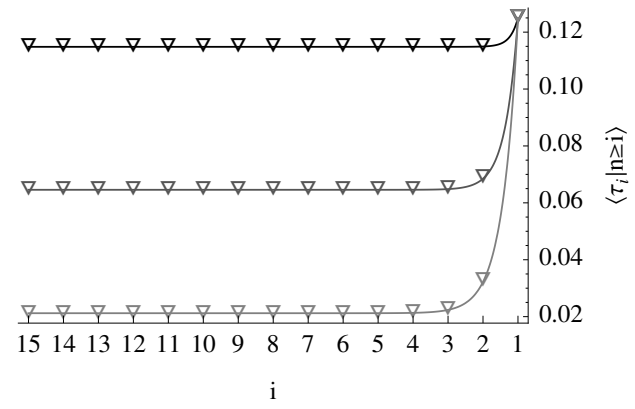

(a) $\lambda_{1}=0.1, \lambda_{2}=0.7, \mu=1$.

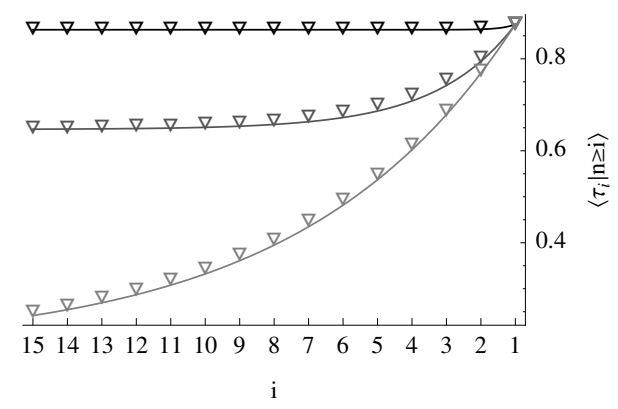

(c) $\lambda_{1}=0.7, \lambda_{2}=0.1, \mu=1$.

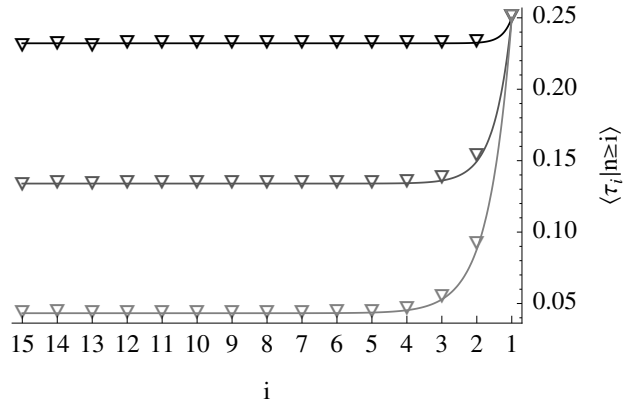

(b) $\lambda_{1}=0.1, \lambda_{2}=0.3, \mu=1$.

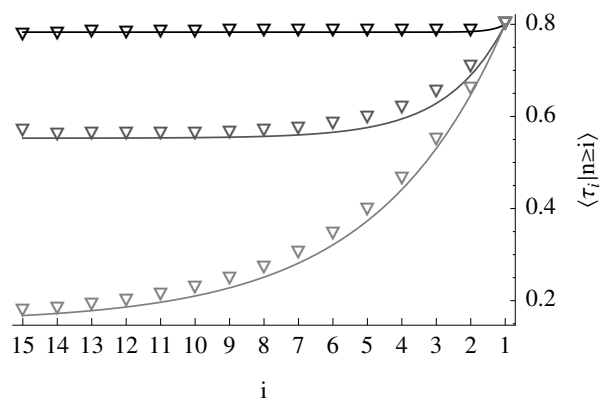

(d) $\lambda_{1}=0.4, \lambda_{2}=0.1, \mu=1$.

Figure 7. Aggregated density profiles scaled by dividing out $(\lambda / \mu)^{i}$. Triangle markers for simulation results, plotted against calculated profile $\left\langle\tau_{i} \mid n \geq i\right\rangle$. For $p=0.1$ (black), $p=1$ (mid-gray), $p=5$ (light gray).

Substituting (88), (92) into (94), Little's result (13) gives the average high priority waiting time,

$$
\bar{W}_{1}=\frac{1}{\lambda_{1}} \bar{N}_{1}=\frac{1}{\lambda_{1}}\left(\alpha \frac{\lambda}{\mu-\lambda}+(1-\alpha) \frac{p \alpha \lambda}{\mu^{2}-p \alpha \lambda}\right) .
$$

With (15), the average low priority waiting time is

$$
\bar{W}_{2}=\frac{1}{\lambda_{2}}\left(\langle n\rangle-\bar{N}_{1}\right)=\frac{1}{\lambda_{2}}(1-\alpha)\left(\frac{\lambda}{\mu-\lambda}-\frac{p \alpha \lambda}{\mu^{2}-p \alpha \lambda}\right) .
$$

Though (95), (96) come from an approximate solution, in the $p \rightarrow 0$ and $p \rightarrow \infty$ limits they give the correct waiting times. Taking first the limit $p \rightarrow 0$, we find

$$
\lim _{p \rightarrow 0} \bar{W}_{1}=\lim _{p \rightarrow 0} \bar{W}_{2}=\frac{1}{\mu-\lambda} .
$$

With $p=0$, high and low priority customers are treated identically. The PEP reduces to a $M / M / 1$ queue with arrival rate $\lambda$ and service rate $\mu$, for which the average waiting time is as given by 97 .

Conversely, if we make the overtake rate infinite, then high priority customers arriving at the queue will immediately overtake any waiting low priority customers. In this limit, high priority customers see an $M / M / 1$ queue with arrival rate $\lambda_{1}$ and service 
rate $\mu$, and indeed we find

$$
\lim _{p \rightarrow \infty} \bar{W}_{1}=\frac{1}{\mu-\lambda_{1}} .
$$

The average waiting time for low priority customers is

$$
\lim _{p \rightarrow \infty} \bar{W}_{2}=\frac{1}{(1-\lambda / \mu)\left(\mu-\lambda_{1}\right)} .
$$

This can be found by directly taking the limit, or via the requirement that $\bar{N}_{1}+\bar{N}_{2}=\langle n\rangle$.

Figure 8 shows $\bar{W}_{1}, \bar{W}_{2}$ plotted as a function $p$. Again we see good agreement between simulation results and the calculated values. Increasing $p$ interpolates between

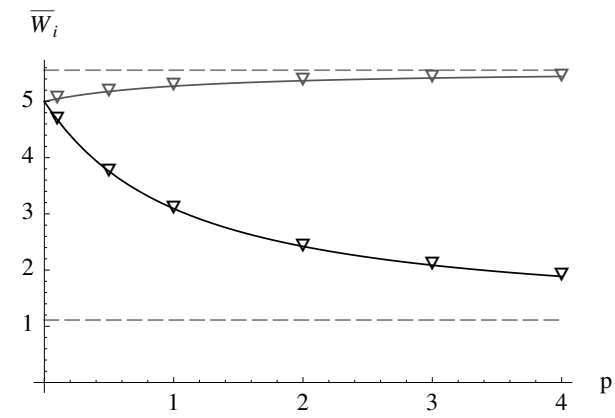

(a) $\lambda_{1}=0.1, \lambda_{2}=0.7, \mu=1$

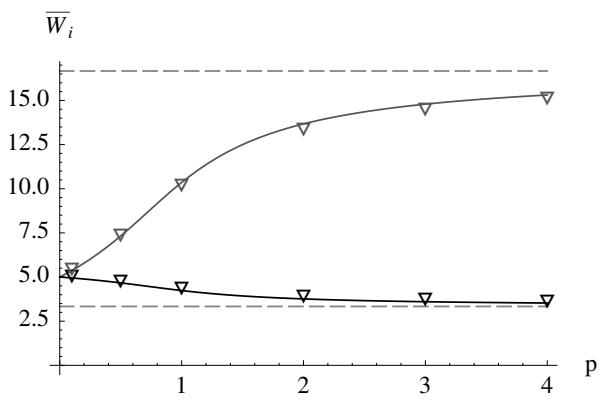

(c) $\lambda_{1}=0.7, \lambda_{2}=0.1, \mu=1$

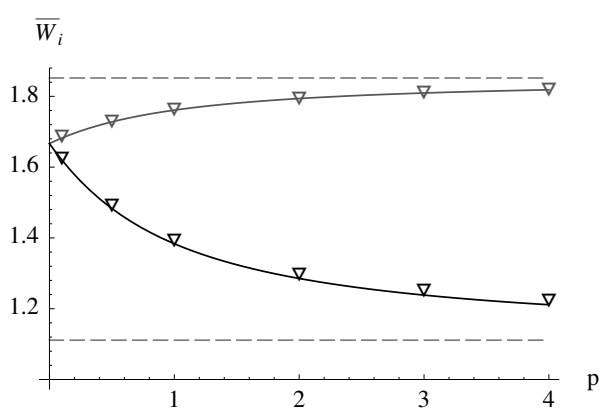

(b) $\lambda_{1}=0.1, \lambda_{2}=0.3, \mu=1$

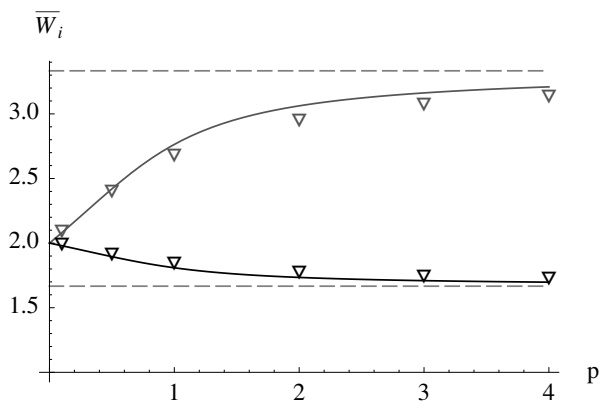

(d) $\lambda_{1}=0.4, \lambda_{2}=0.1, \mu=1$

Figure 8. Average waiting time for high priority customers (black) and low priority customers (gray) plotted against overtake rate $p$. Dashed lines show the asymptotic values.

a first come first served queue $(p=0)$ and strict prioritisation according to customer class $(p \rightarrow \infty)$. In designing a queueing system, one would choose $p$ to give the desired high priority waiting time, within the constraints imposed by the asymptotic limits.

The greatest response in $\bar{W}_{1}$, defined as the maximum value of $\left|\mathrm{d} \bar{W}_{1} / \mathrm{d} p\right|$, occurs at $p=0$ in Figure 8(a), and so there is a strong relative benefit to high priority customers using even small values of $p$. In Figure $8(\mathrm{c})$ the value of the parameters give rise to an inflection point at $p>0$, and hence the largest response in $\bar{W}_{1}$ occurs at some positive value of $p$. A sufficient condition for such an inflection point to occur $\sharp$ is

$\sharp$ This is simpler than trying to solve $\mathrm{d} \bar{W}_{1}^{2} / \mathrm{d} p^{2}=0$. 
$\mathrm{d}^{2} \bar{W}_{1} /\left.\mathrm{d} p^{2}\right|_{p=0}<0$, which happens if and only if

$$
\frac{\lambda_{2}}{\lambda_{1}}<\frac{\lambda_{1}+\lambda_{2}}{\mu} .
$$

Then $\mathrm{d}^{2} \bar{W}_{1} / \mathrm{d} p^{2}$ must change sign as $\lim _{p \rightarrow \infty} \mathrm{d}^{2} \bar{W}_{1} / \mathrm{d} p^{2}>0$. For these values of the parameters the benefit to high priority customers of switching on $p$ is relatively small compared to the penalty for low priority customers.

\section{Conclusion}

In this paper we introduce the prioritising exclusion process: a priority queueing model in which high priority customers are allowed to push ahead in the queue, and thus gain their advantage. The PEP is the exclusion process analog of a well studied priority queueing model, the APQ, a connection which is interesting in itself. But the PEP also has a natural domain wall structure, which allows domain wall dynamics to be derived from the microscopic transition rules. This has recently been achieved for a TASEP with deterministic bulk motion and stochastic boundary conditions [30]. In contrast, the PEP is fully stochastic, but the unique boundary conditions result in the regular behaviour of the jam of high priority customers, the key descriptor in our domain wall model.

The PEP exhibits a phase transition from a phase with finite expected lattice length, to one with an unbounded lattice length. In the unbounded phase, we find the exact solution of the domain wall equations in the $n \rightarrow \infty$ limit, which reveals a further subdivision of this phase into phases with finite or infinite jam length. We find the condition for an infinite jam, in which case low priority customers will, with probability one, never get served. When the jam remains finite we calculate exact stationary density profiles in appropriately defined local reference frames.

In the bounded phase, domain wall theory does not give exact results but leads to two complementary approximate solutions. From a direct application of the domain wall ansatz we find that the shape of the density profile can again be understood in terms of a jam, in this case either localised at the service end or able to grow and fill the lattice. In a second approach, a current conservation equation implies that domain wall theory can be naturally applied to aggregate densities. We thus give very good approximations for these observables and consequently accurately estimate average customer waiting times. We also give the condition for which the stochastic overtaking of low priority customers is most effective as a scheduling mechanism.

The behaviour of the jam of high priority customers plays a key role in understanding all phases of the PEP. There is an interesting analog in the APQ in the notion of accredited customers: class 1 customers with accumulated priority greater than the maximum possible priority of any class 2 customer [2]. This differs from the definition of a jam, since the last accredited customer may be followed by an unaccredited class 1 customer, whereas in the PEP, a jam is always terminated by a class 2 (low 
priority) customer. Understanding this connection may allow us to compute complete waiting time distributions for the PEP, as has already been done for the APQ [2].

\section{Acknowledgement}

We thank Peter Taylor for suggesting the PEP to us as well as for discussions, and Alexandre Lazarescu, Chikashi Arita, Guy Latouce, and Kirone Mallick for discussions and encouragement. We are grateful to the Australian Research Council and the ARC Centre of Excellence for Mathematical Frontiers (ACEMS) for financial support.

\section{References}

[1] Kleinrock L, A delay dependent queue discipline, 1964 Naval Research Logistics Quarterly, 11(3-4) 329-341.

[2] Stanford D A, Taylor P and Ziedins I, Waiting time distributions in the accumulating priority queue, 2014 Queueing Systems 77 297-330.

[3] Hay A M, Valentin E C and Bijlsma R A, Modeling Emergency Care in Hospitals: A Paradox - The Patient Should not Drive the Process, 2006 Proceedings of the 2006 Winter Simulation Conference 439-445.

[4] Spitzer F, Interaction of markov processes, 1970 Advances in Mathematics, 5(2) 246-290.

[5] Liggett T, 1985 Interacting Particle Systems (New York : Springer).

[6] Derrida B, An exactly soluble non-equilibrium system: The asymmetric simple exclusion process, 1998 Physics Reports 301(1-3) 65 - 83.

[7] Schütz G M, Exactly Solvable Models for Many-Body Systems Far from Equilibrium, 2000 Phase Transitions and Critical Phenomena Vol. 19 (London: Academic Press).

[8] Golinelli $\mathrm{O}$ and Mallick $\mathrm{K}$, The asymmetric simple exclusion process: an integrable model for nonequilibrium statistical mechanics, 2006 Journal of Physics A: Mathematical and General 39(41) 12679, arXiv:cond-mat/0611701.

[9] Blythe R A and Evans M R, Nonequilibrium steady states of matrix-product form: a solver's guide, 2007 Journal of Physics A: Mathematical and Theoretical 40(46) R333, arXiv:0706.1678.

[10] Schmittmann B and Zia R K P, Statistical mechanics of driven diffusive systems, 1995 Phase Transitions and Critical Phenomena Vol. 17 (London: Academic Press).

[11] Derrida B, Domany E and Mukamel D, An exact solution of a one-dimensional asymmetric exclusion model with open boundaries, 1992 Journal of Statistical Physics 69(3-4) 667-687.

[12] Schütz G and Domany E, Phase transitions in an exactly soluble one-dimensional exclusion process, 1993 Journal of Statistical Physics 72(1-2) 277-296, arXiv:cond-mat/9303038.

[13] Derrida B, Evans M R, Hakim V and Pasquier V, Exact solution of a $1 D$ asymmetric exclusion model using a matrix formulation, 1993 Journal of Physics A: Mathematical and General 26(7) 1493.

[14] Gwa L-H and Spohn H, Bethe solution for the dynamical-scaling exponent of the noisy Burgers equation, 1992 Phys. Rev. A 46 844-854.

[15] de Gier J and Essler F H L, Bethe ansatz solution of the asymmetric exclusion process with open boundaries, 2005 Phys. Rev. Lett. 95 240601, arXiv:cond-mat/0508707.

[16] Prähofer M and Spohn H, Current fluctuations for the totally asymmetric simple exclusion process, 2002 In and Out of Equilibrium 51, arXiv:cond-mat/0101200

[17] Kolomeisky A B, Schütz G M, Kolomeisky E B and Straley J P, Phase diagram of one-dimensional driven lattice gases with open boundaries 1998 Journal of Physics A: Mathematical and General 31(33) 6911.

[18] Chou T, Mallick K, and Zia R K P, Non-equilibrium statistical mechanics: from a 
paradigmatic model to biological transport, 2011 Reports on Progress in Physics 74(11) 116601, arXiv:1110.1783.

[19] Sugden K E P, Evans M R, Poon W C K, and Read N D, Model of hyphal tip growth involving microtubule-based transport, 2007 Phys. Rev. E 75 031909, arXiv:q-bio/0605013.

[20] Schmitt M and Stark H, Modelling bacterial flagellar growth, 2011 EPL (Europhysics Letters) 96(2) 28001, arXiv:1210.2562.

[21] Dorosz S, Mukherjee S, and Platini T, Dynamical phase transition of a one-dimensional transport process including death, 2010 Phys. Rev. E 81 042101, arXiv:0912.1290.

[22] Johann D, Erlenkämper C, and Kruse K, Length regulation of active biopolymers by molecular motors 2012 Phys. Rev. Lett. 108258103.

[23] Melbinger A, Reese L, and Frey E, Microtubule length regulation by molecular motors, 2012 Phys. Rev. Lett. 108 258104, arXiv:1204.5655.

[24] Yanagisawa D, Tomoeda A, Jiang R, and Nishinari K, Excluded volume effect in queueing theory, 2010 JSIAM Letters 2 61-64, arXiv:1001.4124.

[25] Arita C Queueing process with excluded-volume effect, 2009 Phys. Rev. E 80051119 , arXiv:0911.2528.

[26] Arita C and Schadschneider A, Density profiles of the exclusive queuing process, 2012 Journal of Statistical Mechanics: Theory and Experiment P12004, arXiv:1210.1482.

[27] Sugden K E P and Evans M R, A dynamically extending exclusion process, 2007 Journal of Statistical Mechanics: Theory and Experiment 2007(11) P11013, arXiv:0707.4504.

[28] Nowak S A, Fok P-W and Chou T, Dynamic boundaries in asymmetric exclusion processes 2007 Phys. Rev. E 76 031135, arXiv:0708.0259.

[29] Arita C and Schadschneider A, Critical behavior of the exclusive queueing process, 2013 EPL (Europhysics Letters) 104(3) 30004, arXiv:1308.2417.

[30] Cividini J, Hilhorst H J and Appert-Rolland C, Exact domain wall theory for deterministic TASEP with parallel update, 2014 J. Phys. A: Math. Theor. 47 222001, arXiv:1310.2090.

[31] Kleinrock L, 1976 Queueing Systems, Volume II: Computer Applications (Wiley Interscience).

[32] Kleinrock L, 1975 Queueing Systems, Volume I: Theory (Wiley Interscience).

[33] Santen L and Appert C, The asymmetric exclusion process revisited: Fluctuations and dynamics in the domain wall picture, 2002 Journal of Statistical Physics 106(1-2) 187-199, arXiv:condmat/0107238.

[34] Popkov V, Santen L, Schadschneider A and Schütz G M, Empirical evidence for a boundary-induced nonequilibrium phase transition, 2001 Journal of Physics A: Mathematical and General 34(6) L45.

[35] Cook L J and Zia R K P, Feedback and fluctuations in a totally asymmetric simple exclusion process with finite resources, 2009 Journal of Statistical Mechanics: Theory and Experiment 2009(02) P02012, arXiv:0811.1543.

[36] de Gier J, Finn C, and Sorrell M, The relaxation rate of the reverse-biased asymmetric exclusion process, 2011 Journal of Physics A: Mathematical and Theoretical 44(40) 405002, arXiv:1107.2744. 